\title{
Piezoresistive cantilever designed for torque magnetometry
}

\author{
M. Willemin \\ Physik-Institut, Universität Zürich, Winterthurerstrasse 190, CH-8057 Zürich, Switzerland \\ and IBM Research Division, Zurich Research Laboratory, CH-8803 Rüschlikon, Switzerland \\ C. Rossel, ${ }^{\text {a) }}$ J. Brugger, M. H. Despont, H. Rothuizen, and P. Vettiger \\ IBM Research Division, Zurich Research Laboratory, CH-8803 Rüschlikon, Switzerland \\ J. Hofer \\ Physik-Institut, Universität Zürich, Winterthurerstrasse 190, CH-8057 Zürich, Switzerland \\ and IBM Research Division, Zurich Research Laboratory, CH-8803 Rüschlikon, Switzerland \\ H. Keller \\ Physik-Institut, Universität Zurich, Winterthurerstrasse 190, CH-8057 Zürich, Switzerland
}

(Received 21 July 1997; accepted for publication 29 October 1997)

\begin{abstract}
New piezoresistive silicon cantilevers designed specifically for torque magnetometry on microscopic samples have been microfabricated and tested. These levers have been optimized to detect the torque in two directions corresponding to flexion and torsion. Torque resolution of $\sim 10^{-14} \mathrm{~N}$ m can be achieved depending on the operating mode. In one version an integrated loop allows an absolute calibration of the device with an accuracy of $\sim 1 \%$. This loop can also be used to excite the lever mechanically. One application is the determination of the mass of nanogram samples by measuring the resonance frequency shift (nanobalance). (C) 1998 American Institute of Physics. [S0021-8979(98)09203-2]
\end{abstract}

\section{INTRODUCTION}

The fabrication of micromechanical cantilevers has given rise to numerous powerful applications. The new generation of microlevers is generally made of silicon, silicon oxide, silicon nitride, or gallium arsenide, ${ }^{1}$ and the precise measurement of their displacement is done by optical ${ }^{2,3}$ or integrated piezoresistive, ${ }^{4,5}$ piezoelectric, ${ }^{6}$ or capacitive techniques. $^{7}$ Aside from their general use in scanning force microscopies (SFM), microlevers can be applied as different types of physical or chemical sensors. Of the many examples, one can cite their use for the mechanical detection of magnetic torque, of magnetic resonance, or their application as temperature sensors, gas sensors, calorimeters, or spectrometers. ${ }^{8}$ The small size and mass of these devices in addition to their ultrahigh sensitivity allow very short response times. We have shown that piezoresistive Si cantilevers are nicely suited for torque magnetometry on micrometer-sized superconducting or magnetic samples. ${ }^{9,10}$ The torque magnetometer is complementary to a superconducting quantum interference device (SQUID) for measuring the magnetization $M$ or susceptibility $\chi$ as a function of magnetic field and temperature. Besides its improved sensitivity compared to that of commercial SQUID magnetometers, it allows us to measure in a simple way the anisotropy constants of magnetic materials plunged into a homogeneous applied field $\vec{B}$. The torque generated by $\vec{B}$ on the sample of magnetic moment $\vec{m}$ is given by $\vec{\tau}=\vec{m} \times \vec{B}$. The induced rotation of the sample fixed on the free end of the cantilever generates a deflection, which can be measured with high precision. The above expression naturally requires the sample to be anisotropic, otherwise $\tau$ would be zero. Fortunately most

a)Electronic mail: rs1@zurich.ibm.com magnetic materials (e.g., ferro- or ferrimagnets, superconductors) are intrinsically anisotropic owing to their crystallographic symmetry. From the angular-dependent torque data, $\tau(\theta)$, the anisotropy energy, and the axes of easy magnetization can be determined.

Earlier versions of torquemeters such as the torsion pendulum ${ }^{11}$ and the conventional capacitance torquemeter ${ }^{12-14}$ were rather large in size and thus suited only for millimeter-sized samples or films. Moreover, their large dimensions and mass made them sensitive to external perturbations (e.g., vibrations, air flow, and temperature gradients) and consequently more delicate to manipulate with a minimum of background noise. Our purpose here is to continue the development of cantilevers not only to improve their sensitivity but also to make them more practical and versatile, for example, by rendering them almost equally sensitive to flexion and torsion and by integrating in them a current loop for calibration and activation purposes.

\section{DESIGN AND CALCULATION}

The torquemeters presented here (Figs. 1 and 2) are microscopic silicon piezoresistive cantilevers. These sensors are designed to detect a torque with respect to two axes (Fig. 1). In other words, the levers can work either in flexion or torsion mode. In the past, calibration of a torquemeter using standard microcantilevers required the use of a superconducting sample in the Meissner state and with well-known geometry. ${ }^{9}$ Therefore it is useful to find a simple and convenient absolute calibration method that is sample-independent.

The design is based on standard two-leg flexion cantilevers. ${ }^{4}$ The advantages of initiating a design with this geometry are simplicity, robustness, and high sensitivity. Mounting a sample on the platform of such a cantilever is 


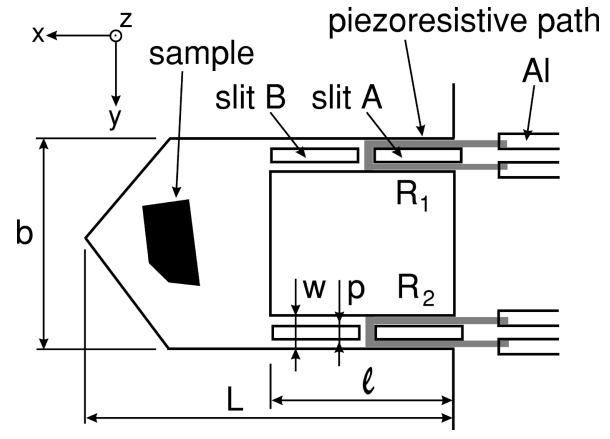

FIG. 1. Schematic view of the silicon piezoresistive two-leg cantilever for ultrasensitive torque magnetometry. The slits in both legs are to optimize sensitivity and to define the piezoresistive path. The lever dimensions are summarized in Table I.

particularly convenient. Moreover, the present geometry allows the lever to be used as an atomic force microscopy (AFM) sensor.

As mentioned above, the geometry of a very sensitive torque sensor must be chosen such that it keeps the effect of undesirable external accelerations below the resolution limit. One solution to this inertial problem is to reduce the masses involved as much as possible. Another approach ${ }^{15}$ is to forsake the standard flexible beam concept and design devices in favor of a more complicated geometry, for example a geometry based only on torsion arms. For the two torquemeters presented here (Figs. 1 and 2), the first solution was considered by minimizing the mass of the device. In this case, only accelerations greater than $0.1 \mathrm{~ms}^{-2}$ (seldom exceeded in a laboratory situation) would disturb a measurement.

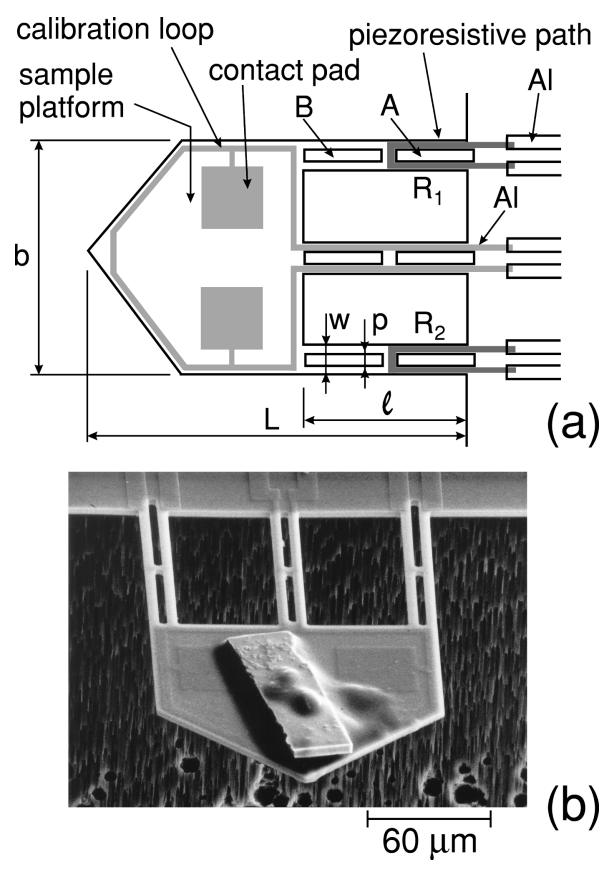

FIG. 2. (a) Schematic view of the three-leg cantilever with integrated loop for calibration. Lever dimensions are summarized in Table I. (b) SEM image of the cantilever with single crystal of a high-temperature superconducting cuprate.

\section{A. Two-leg lever}

We propose here a simple design with two legs, each holding a piezoresistive element (Fig. 1). A torque applied to the cantilever will produce a mechanical stress in each leg, which can be detected by a change of the two piezoresistances $R_{1}$ and $R_{2}$. The torque $\tau_{x}$ for torsion or $\tau_{y}$ for flexion can be extracted by measuring $\Delta\left(R_{1}-R_{2}\right)$ or $\Delta\left(R_{1}+R_{2}\right)$, respectively. The relative change in piezoresistance due to a mechanical stress is given by

$$
\frac{\Delta R}{R}=\beta \pi_{L} \overline{\sigma_{p}}
$$

where $\pi_{L}$ is the longitudinal piezoresistive coefficient, which is significantly dependent on doping. ${ }^{16,17}$ The parameter $\overline{\sigma_{p}}$ is the average elastic stress at the surface of the mechanical element where the piezoresistor is located. The term $\beta$ is a geometrical correction of the finite thickness of the piezoresistive path. For a first calculation, we used the following parameters: $\beta \approx 0.8$ and $\pi_{L} \approx 4.5 \times 10^{-10} \mathrm{~m}^{2} \mathrm{~N}^{-1}$. 4,16 These quantities are subject to some fluctuations related to the microfabrication process.

The use of two piezoresistors allows torque detection about the two axes $x$ and $y$; it would be ideal to have exactly the same torque sensitivity for both directions. This condition is not easy to fulfill in the actual lever geometry and for realistic dimensions because the flexion mode is always more sensitive. In our case, for a torque $\tau_{y}$, the lever works in the flexion mode and both legs are submitted to a pure flexion stress. On the other hand, for a torque $\tau_{x}$, in the so-called torsion mode, torsion and flexion are generated in each leg. As shown in Fig. 1, the legs of the cantilever have been designed with two slits (A and $\mathrm{B}$ ). The role of the A-slits is to insulate electrically the in and out-going piezoresistive paths. The role of the B-slits is to provide a symmetrical deformation of the leg in the torsion mode, while increasing its sensitivity and reducing the inertial moment. With this design, the current through the piezoresistive paths does not significantly perturb the torque detection by inducing Lorentz forces. This effect is negligible for two reasons: the piezoresistive paths along the $y$-direction are very short and all paths along the $x$-direction form pairs with opposite currents for optimal compensation. A peculiarity of the flexion due to a torque $\tau_{y}$ is that the ratio $\Delta R / R$ is independent of the length of the piezoresistive path, because $\sigma_{p}$ remains constant along the entire leg. However, if the flexion is due not to a torque but to a force, as in AFM techniques for example, $\sigma_{p}$ varies linearly along the leg and reaches its maximum value at the basis of the lever. In this case, the average elastic stress $\overline{\sigma_{p}}$ depends on the length of the piezoresistor. Because the lever is primarily designed for torque magnetometry, we prefer to optimize the length of the piezoresistive paths by considering the torsion due to a torque $\tau_{x}$. In torsion mode, $\Delta R / R$ decreases over the length of the path and vanishes when the path covers the entire leg because $\overline{\sigma_{p}}=0$ in this limit, Eq. (1). The value of $\overline{\sigma_{p}}$ would be maximized for infinitely small paths. Obviously, this is not possible because $R$ would vanish. A good compromise is to choose the path length to be half that of the legs, so that 
TABLE I. Typical dimensions of the two versions of microfabricated piezoresistive cantilevers.

\begin{tabular}{lcc}
\hline \hline \multicolumn{1}{c}{ Lever } & two legs & three legs \\
\hline$L$ (lever length) $(\mu \mathrm{m})$ & 200 & 240 \\
$l$ (leg length) $(\mu \mathrm{m})$ & 100 & 100 \\
$w$ (leg width) $(\mu \mathrm{m})$ & 10 & 10 \\
$b$ (lever width) $(\mu \mathrm{m})$ & 117 & 143 \\
$p$ (slit width) $(\mu \mathrm{m})$ & 4 & 4 \\
$t$ (thickness) $(\mu \mathrm{m})$ & 4 & 4 \\
\hline \hline
\end{tabular}

$\overline{\sigma_{p}}=\sigma_{p}^{\max } / 2$. For the given geometry (Fig. 1) in flexion mode, the averaged stress over the piezoresistive path becomes

$$
\overline{\sigma_{p}^{f}}=\frac{3 \tau_{y}}{(w-p) t^{2}},
$$

where $t$ is the lever thickness. In torsion mode, the corresponding stress is

$$
\overline{\sigma_{p}^{t}}=\frac{3}{2} \tau_{x}\left(\frac{b(w-p) t^{2}}{l}+\frac{G l}{E b}\left[\frac{\left(w^{3}-p^{3}\right)+(w-p) t^{2}}{3}\right]\right)^{-1},
$$

where $E$ is the Young's modulus and $G$ the shear modulus of $\langle 110\rangle \mathrm{Si}^{18,19}$ The lever dimensions are defined in Fig. 1. In order to reach the same sensitivity in torsion and flexion, the following condition must be satisfied:

$$
\frac{\overline{\sigma_{p}^{f}}}{\tau_{y}}=\frac{\overline{\sigma_{p}^{t}}}{\tau_{x}} .
$$

According to Eq. (2), the dimensions $t$ and $(w-p)$ should be chosen as small as possible to optimize the sensitivity in the flexion mode. The choice of these dimensions is also governed by the optimization in torsion mode. With the values of $t, w$, and $p$ given in Table I, the levers are sufficiently robust and their dimensions compatible with the technology used for microfabrication. In principle, the optimization of the torsion mode with Eq. (4) should yield the ratio of the two other lever dimensions $b / l$. In fact this is not easily obtained and, for realistic values of $t$ and $(w-p)$, only a complex solution can be found. The solution always leads to a smaller sensitivity in torsion than in flexion:

$$
\frac{\overline{\sigma_{p}^{t}}}{\tau_{x}}<\frac{\overline{\sigma_{p}^{f}}}{\tau_{y}}
$$

With a global choice of the lever dimensions $t, w, p, b$, and $l$, the optimized sensitivity ratio closest to 1 that can be obtained is

$$
\left(\overline{\frac{\sigma_{p}^{f}}{\tau_{y}}}\right)\left(\overline{\frac{\sigma_{p}^{t}}{\tau_{x}}}\right)^{-1}=4.6
$$

The dimensions of this two-leg cantilever are summarized in Table I. Note that the values of $\beta, \pi_{L}$, and the lever length $L$ are irrelevant for this optimization.

\section{B. Three-leg lever}

A second version of the cantilever discussed above was designed with a small coil integrated on the platform (Fig. 2)
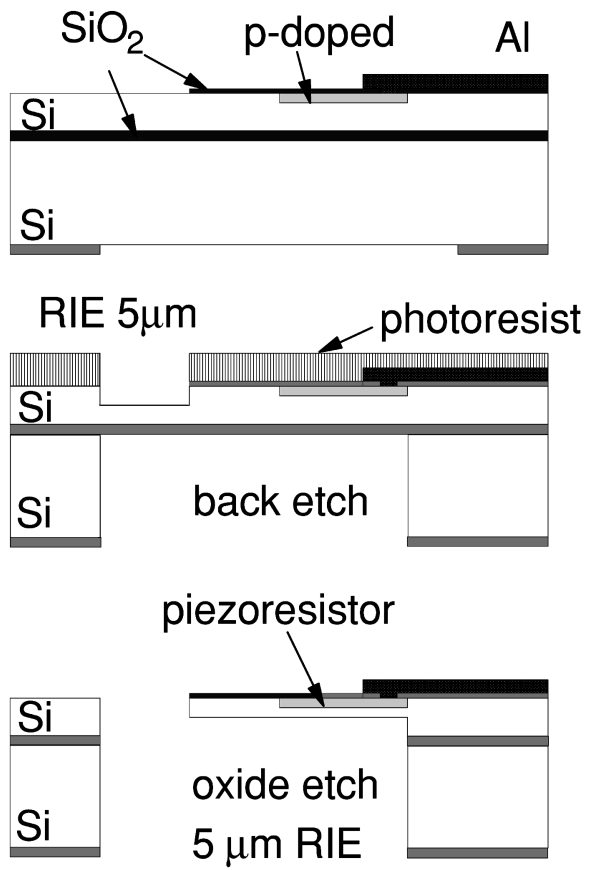

FIG. 3. Sequences of the microfabrication process.

to allow absolute calibration. An additional central leg is attached to hold the metallic paths leading to the calibration loop. To reduce the influence of this central leg on the lever properties, we also designed it with two slits. After performing the same optimization as for the two-leg cantilever, a sensitivity ratio of about 3.8 is reached (see appendix). This value is slightly better than that for the two-leg lever because the addition of a third leg reduces the sensitivity to flexion more than to torsion. The dimensions of the three-leg cantilever are summarized in Table I.

The calibration loop has an area of $S=1.2 \times 10^{-8} \mathrm{~m}^{2}$. We chose a metallic path thickness of $300 \mathrm{~nm}$ of Al to minimize the bilayer effect (owing to the different thermal expansion of $\mathrm{Al}$ and $\mathrm{Si}$ ) yet still keep the resistance below $100 \Omega$. A current of $1 \mathrm{~mA}$ produces a measurable magnetic moment of $m=1.2 \times 10^{-11} \mathrm{~A} \mathrm{~m}^{2}$, while retaining a reasonable power dissipation of $0.1 \mathrm{~mW}$. Two additional contact pads are deposited on the lever platform [Fig. 2(a)]. By breaking the calibration coil between the pads, two electrical contacts can be used for resistance measurements on the sample.

For both cantilevers, the platform end has been designed with a triangular shape and a tip suitable for AFM applications.

\section{MICROFABRICATION}

The microfabrication ${ }^{20}$ of the piezoresistive sensors is sketched in Fig. 3 and can be summarized as follows. The process sequence starts by defining alignment marks on a 10$\mu \mathrm{m}$-thick, slightly $n$-doped, polished (100) silicon membrane that is separated from the substrate by a $1-\mu \mathrm{m} \mathrm{SiO}_{2}$ layer. Local ion implantation and an annealing step define the $p^{+}$-doped piezoresistive sensing paths that are oriented

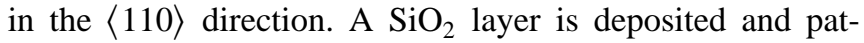
terned to separate the subsequent metallization from the 


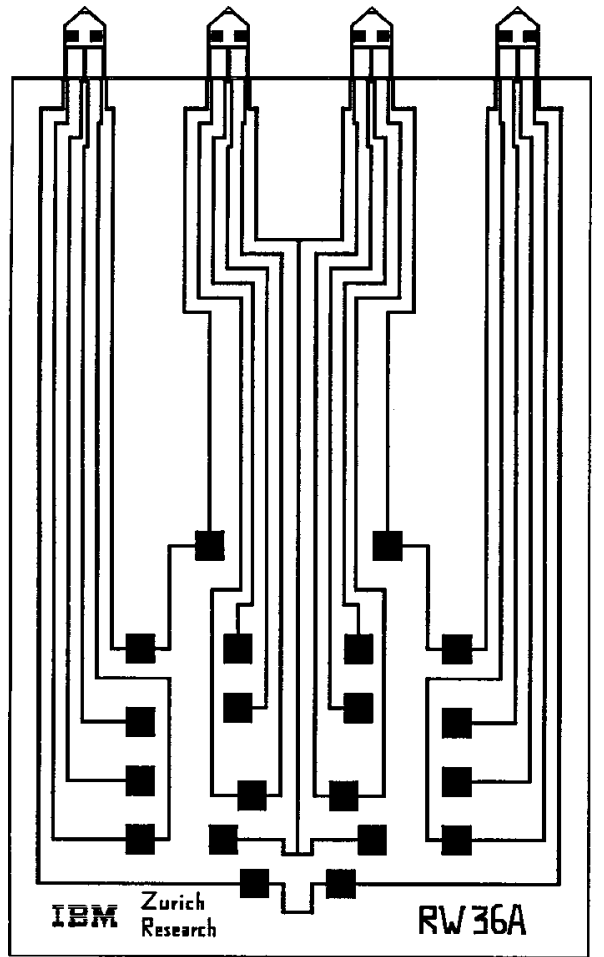

FIG. 4. Schematic view of the entire chip with the four identical three-leg levers. The contact padding allows the levers to be connected in various configurations.

membrane. Only small contact holes in this $\mathrm{SiO}_{2}$ layer allow the connection between the piezoresistive path and the metallic wires. The lever is then structured by using etching techniques from both wafer sides, which simultaneously defines the chip geometry. The lever thickness is very homogeneous owing to the buried oxide layer, which serves as an etch stop from the back side. After removing the $\mathrm{SiO}_{2}$, the levers are reduced to the desired thickness in a final dry etching step from the back side. Figure 4 shows the layout of the entire chip containing four identical three-leg levers.

\section{EXPERIMENTAL DETAILS}

\section{A. Measuring circuit}

In order to detect very small changes in the piezoresistances $R_{1}$ and $R_{2}$, we use a conventional Wheatstone bridge. To detect $\tau_{y}$, corresponding to the flexion mode, the configuration measuring $\Delta\left(R_{1}+R_{2}\right)$ is required [Fig. 5(a)]. In

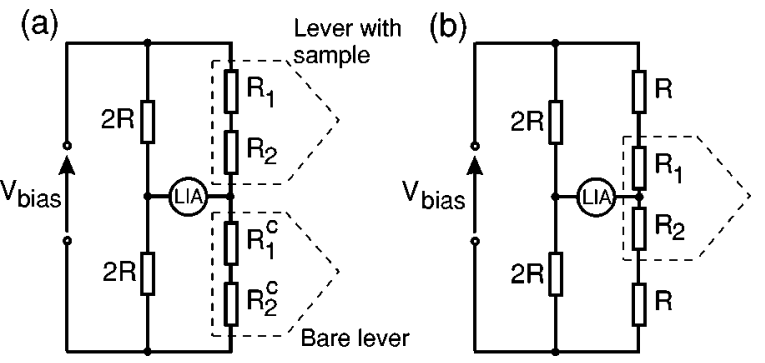

FIG. 5. Wheatstone bridge configurations for (a) flexion mode and (b) torsion mode measurements. the torsion mode, $\tau_{x}$ is measured via $\Delta\left(R_{1}-R_{2}\right)$, using the second circuit shown in Fig. 5(b).

For each of these two modes, moreover, we distinguish between two different regimes. In the so-called static regime, $\Delta R / R$ (corresponding to a quasistatic torque) is measured by a lock-in technique using the modulated bias voltage $V_{\text {bias }}$ as reference. In the second case, the lock-in detection is in phase with the oscillating torque signal, which sets the lever in a vibration or dynamic regime. According to the relation $\vec{\tau}=\vec{m} \times \vec{B}$, this torque signal can be generated either by an oscillating magnetic field $B(t)$ or by a modulated magnetic moment $m(t)$ (e.g., a calibration loop). The great advantage of the dynamic regime, for flexion or torsion, is that it precludes resistance drifts, unlike the static regime. Temperature dependence and magnetoresistance of the piezoresistors are unavoidable. ${ }^{9}$ These undesirable effects are minimized in a differential mode using a second reference cantilever [Fig. $5(\mathrm{a})$ ] involving the compensation resistances $R_{1}^{c}$ and $R_{2}^{c}$. However drifts can never be fully compensated owing to irregularities in microfabrication, but a reduction by a factor of at least 10 can usually be achieved. In the dynamic regime we found a resolution in torque that is 100 times better than in the corresponding static regime (see Sec. IV B).

To avoid heating effects, the bias voltage for all configurations is limited to $1 \mathrm{~V}_{\mathrm{rms}}$.

\section{B. Calibration and resolution}

In general, the absolute calibration of a microtorquemeter can be difficult. Thanks to the high sensitivity of our design, only a well-known, small magnetic moment is necessary to perform a good calibration. A loop with one turn is sufficient (see Sec. II) to achieve calibrations with an accuracy of about $1 \%$ in flexion and in torsion modes. Both static and dynamic regimes are well suited for a calibration. This method has the advantage of being independent of the sample mounted on the lever. We present a calibration performed in the dynamic torsion mode [Fig. 6(a)] at room temperature as an example. We chose a magnetic field $B=0.5 \mathrm{~T}$ and a current in the loop $I_{\text {loop }}=0.707 \mathrm{~mA}_{\text {rms }}$ at $4 \mathrm{kHz}$. The corresponding magnetic moment is $m=I_{\text {loop }} \cdot S=0.849$ $\times 10^{-11} \mathrm{~A}_{\mathrm{rms}} \mathrm{m}^{2}$, where $S$ is the area of the loop. In this case, the torque is well fitted with $\sin (\theta)$, where $\theta$ is the angle between $\vec{m}$ and $\vec{B}$. The maximum torque signal expressed in terms of the voltage measured on the Wheatstone bridge [Fig. 5(b)] is $U_{\max }=1.50 \mu \mathrm{V}_{\mathrm{rms}}$. The corresponding torque $\left(\theta=-90^{\circ}\right)$ is given by $\tau_{\max }=m B=4.25 \times 10^{-12} \mathrm{Nm}$ $\mathrm{rms}$. The resulting calibration factor for the torsion mode is $2.83 \mathrm{pNm} / \mu \mathrm{V}$. The linearity in magnetic field [Fig. 6(b)] and in current [Fig. 6(c)] are evident. Exactly the same type of calibration in dynamic flexion mode yields a factor of 1.36 $\mathrm{pNm} / \mu \mathrm{V}$. The 2.1 ratio of these two factors is different from the calculated sensitivity ratio of 3.8 obtained for this specific design. Small mismatches between effective and calculated dimensions of the lever explain this discrepancy. Indeed, we found from scanning electron microscopy (SEM) images that the effective lever thickness is closer to $5 \mu \mathrm{m}$ than to the $4 \mu \mathrm{m}$ used in our calculations. Taking the value of $5 \mu \mathrm{m}$, we obtained a theoretical sensitivity ratio of 3.1 . 

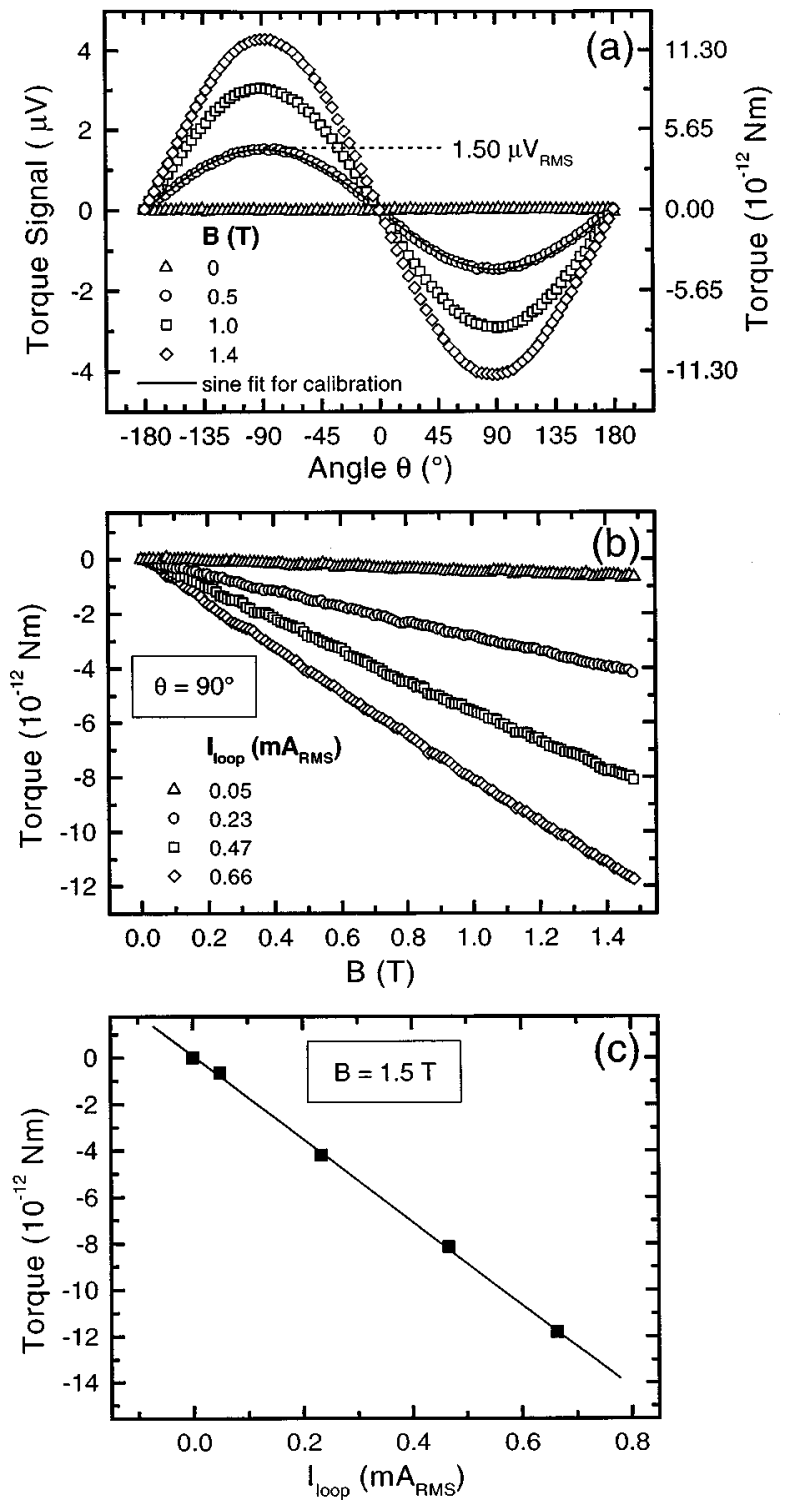

FIG. 6. Measurements with the calibration loop. (a) Angular-dependent torque $\tau(\theta)$, where $\theta$ is the angle between the magnetic moment $\vec{m}$ produced by the loop and the magnetic field $\vec{B}$. The calibration parameter is extracted from these curves and applied to the next two diagrams. (b) Magnetic field-dependent torque $\tau(B)$ at fixed angle $\theta=90^{\circ}$. (c) Currentdependent torque $\tau\left(I_{\text {loop }}\right)$ at fixed magnetic field $\vec{B}$.

Furthermore, small deviations of the legs' geometry as a result of etching are inevitable and can modify the sensitivity ratio significantly, which underscores the importance of an absolute calibration. The inevitable temperature dependence of the piezoresistive coefficient $\pi_{L}$ and of the resistance itself also affect the sensitivity. ${ }^{16}$

From the voltage noise level observed during the calibration process, the typical resolution of the device can be estimated. In the dynamic regime, the resolution is about $10^{-14} \mathrm{Nm}$, whereas for the static regime it reaches $10^{-12}$ $\mathrm{Nm}$. The dynamic resolution is nearly time-independent, but in the static regime the time scale must be indicated. We consider here a typical measurement time of about $120 \mathrm{~s}$ and a lock-in integration constant less than $100 \mathrm{~ms}$. The above resolutions are comparable to those obtained with Park Sci-

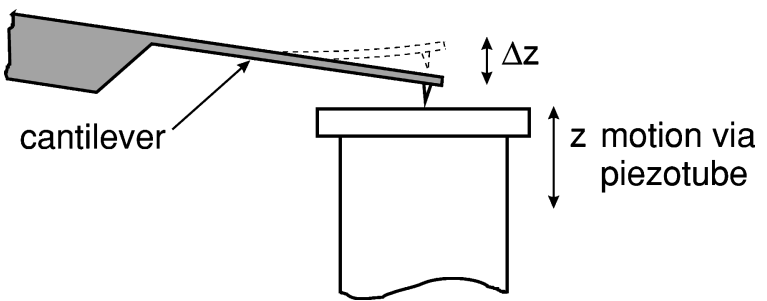

FIG. 7. Calibration of the displacement $\Delta z$ by use of an AFM scanner.

entific Instrument levers, ${ }^{9}$ for which we assumed a measurable lever deflection of about $0.01 \mathrm{~nm}$ as reported by Tortonese et al. ${ }^{4}$ for the dynamic regime. The resolutionlimiting factor in the static regime is the time fluctuation of the piezoresistances (intrinsic noise).

The product $\beta \pi_{L}$ can be estimated with the calibration loop. From the Wheatstone bridge configurations (Fig. 5) one can write

$$
\Delta V=\frac{V}{4 R} \Delta R=\frac{V}{4} \beta \pi_{L} \overline{\sigma_{p}} .
$$

For the flexion mode and substituting the expression for $\overline{\sigma_{p}^{f}}$ adapted for the three-leg cantilever (see appendix) we obtain

$$
\beta \pi_{L}=\frac{\Delta V}{\tau_{y}} \frac{2(w-p) t^{2}}{V} .
$$

In the case of a 5- $\mu \mathrm{m}$-thick lever, taking $V=1 \mathrm{~V}_{\text {rms }}$ and the calibration factor $\tau_{y} / \Delta V=1.36 \mathrm{pNm} / \mu \mathrm{V}$, we obtain a value of $\beta \pi_{L} \approx 2.2 \times 10^{-10} \mathrm{~m}^{2} / \mathrm{N}$ at room temperature, which is consistent with values of the piezoresistive coefficient $\pi_{L}$ and of the geometrical factor $\beta$ found in the literature. ${ }^{4}$ Henceforth we will use this value.

As mentioned above, these devices can also be used for AFM. For this application, the link between the displacement of the lever's tip and the relative change of the piezoresistance is the parameter of interest. Use of a calibrated AFM scanner (Nanoscope) is appropriate to obtain the calibration factor in the AFM mode (Fig. 7), which can also be calculated from the elastic and piezoresistive properties. For the example of a two-leg lever, the AFM calibration factor is

$$
\frac{\Delta R}{R \Delta z}=\beta \pi_{L} \frac{3(4 L-l) E t}{8\left[L^{3}-(L-l)^{3}\right]} .
$$

We have assumed here that, when considering the displacement $\Delta z$ of a lever under flexion, the platform remains rigid and only the legs are flexed (no torsion mode). ${ }^{9}$ A summary of the calculated and measured calibration factors is given in Table II. For the theoretical results we assumed $\beta \pi_{L} \approx 2.2 \times 10^{-10} \mathrm{~m}^{2} / \mathrm{N}$, a Young's modulus of $E=170 \mathrm{GPa}$ and a Poisson's ratio of 0.28 . Measured values are in parentheses. The observed mismatches are normal for this kind of microfabrication and are due primarily to geometrical dispersion.

\section{Resonance}

Another application of the integrated loop is to excite the lever. By working in a constant magnetic field, the oscilla- 
TABLE II. Calibration factor and piezosensitivity to flexion (torque $\tau_{y}$ ) and torsion (torque $\tau_{x}$ ) calculated and measured (in parentheses) for the two versions of piezoresistive cantilevers. In addition, the calculated piezosensitivity to a displacement $\Delta z$ of the free end of the levers is compared to that measured with a calibrated AFM scanner.

\begin{tabular}{|c|c|c|}
\hline Lever sensitivity & two legs & three legs \\
\hline Flexion & 0.59 & 0.87 \\
\hline$\Delta \tau_{y}(\mathrm{pNm})$ & & $(1.36)$ \\
\hline $\begin{array}{l}\overline{\Delta V}(\overline{\mu \mathrm{V}}) \\
\text { in other units }\end{array}$ & $6.7 \times 10^{-6}$ & $4.6 \times 10^{-6}$ \\
\hline$\frac{\Delta R}{R \Delta \tau_{y}}(\mathrm{pNm})^{-1}$ & & $\left(2.94 \times 10^{-6}\right)$ \\
\hline Torsion & 2.73 & 3.3 \\
\hline$\Delta \tau_{x}(\mathrm{pNm})$ & & $(2.83)$ \\
\hline $\begin{array}{l}\overline{\Delta V}(\overline{\mu \mathrm{V}}) \\
\text { in other units } \\
\frac{\Delta R}{R \Delta \tau_{x}}(\mathrm{pNm})^{-1}\end{array}$ & $1.5 \times 10^{-6}$ & $\begin{array}{c}1.2 \times 10^{-6} \\
\left(1.41 \times 10^{-6}\right)\end{array}$ \\
\hline Sensitivity ratio & 4.6 & $\begin{array}{l}3.8 \\
(2.1)\end{array}$ \\
\hline AFM & $5.6 \times 10^{-6}$ & $4.3 \times 10^{-6}$ \\
\hline$\frac{\Delta R}{R \Delta z}(\mathrm{~nm})^{-1}$ & $\left(4.6 \times 10^{-6}\right)$ & $\left(3.9 \times 10^{-6}\right)$ \\
\hline
\end{tabular}

tions are controlled by the frequency of an ac current in the loop. Thus it is possible to measure very precisely the resonance frequency of the lever. For this measurement the bias voltage of the Wheatstone bridge is maintained constant $\left(1 \mathrm{~V}_{\mathrm{dc}}\right)$ and a lock-in detection of the output signal in phase with the loop current is performed.

The cantilever can also be used as a microbalance. The sample mass $m_{e}$ is simply given by the resonance frequency shift compared to the bare lever:

$$
m_{e}=\frac{k}{(2 \pi)^{2}}\left(\frac{1}{\nu^{2}}-\frac{1}{\nu_{0}^{2}}\right) .
$$

The spring constant of the lever $k$ can be determined from the expression of the AFM calibration factor, ${ }^{9}$ Eq. (9),

$$
k=\frac{F}{\Delta z}=\alpha \frac{(w-p) E t^{3}}{2\left[L^{3}-(L-l)^{3}\right]},
$$

where $\alpha=1$ and 3/2 for the two-leg and three-leg lever, respectively. This expression is correct only if the external force $F$ is applied at the lever extremity and will be wrong for determination of the resonance of the bare lever: $\nu_{0}=(1 / 2 \pi)(\mathrm{k} / \mathrm{m})^{-1 / 2}$. It is necessary to consider a spring constant for a force applied approximatively at the platform center. Thus $L$ must be substituted by $(L+l) / 2$ in the above expression of $k$. Taking the density of Si to be $\rho=2.34 \times 10^{3}$ $\mathrm{kg} / \mathrm{m}^{3}$, a lever thickness of $t=5 \mu \mathrm{m}$ and a platform area of about $1.5 \times 10^{-8} \mathrm{~m}^{2}$ [note that the platform area is slightly larger than the loop (Fig. 2)], the platform mass and the spring constant can be calculated to be $m \approx 1.8 \times 10^{-10} \mathrm{~kg}$ and $k \approx 20.9 \mathrm{~N} / \mathrm{m}$, respectively. These values yield a lever eigenfrequency of $\nu_{0} \approx 54 \mathrm{kHz}$. In order to determine a mass with this method, we used as test sample a small single crystal of the high-temperature superconductor $\mathrm{HgBa}_{2} \mathrm{CuO}_{4+\delta}$ (Hg-1201), which has a critical temperature of $T_{c}=91 \mathrm{~K}$.

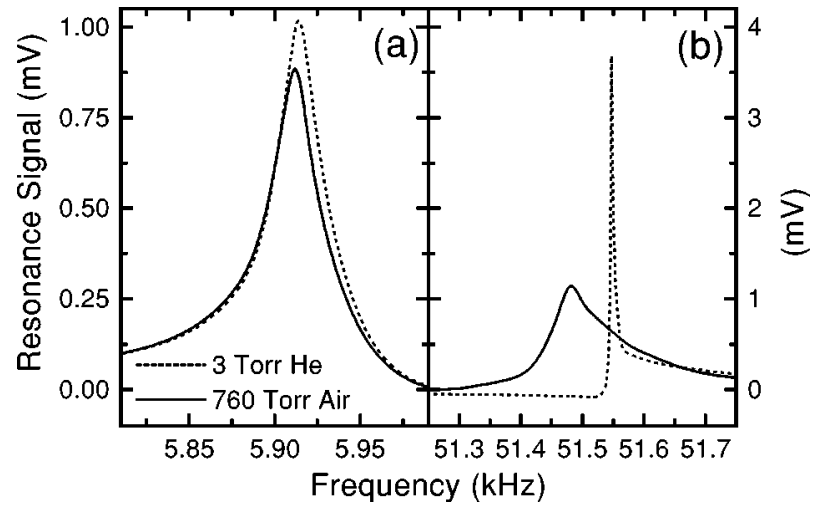

FIG. 8. Resonance curves of three-leg lever at room temperature (a) with sample, (b) without sample. The mechanical excitation is produced by an ac current in the calibration loop at fixed field $\vec{B}$. The mass of the sample is derived from the frequency shift. Under reduced gas pressure the resonance shifts to higher frequency and its amplitude increases.

This same sample will also be used in another measurement to be discussed in Sec. IV D. From the resonance curves measured at room temperature and in reduced He gas pressure (3 Torr), see Fig. 8, we obtained the resonance frequency $\nu \approx 5.913 \mathrm{kHz}$ with sample and $\nu_{0} \approx 51.5 \mathrm{kHz}$ without sample. The latter experimental value is in very good agreement with the calculated one. The sample was fixed on the platform with a small quantity of grease. The mass of the sample and grease determined from the frequency shift is $m_{e} \approx 14.9 \mu \mathrm{g}$. The mass determination would be more accurate if the resonance frequency $\nu_{0}$ were measured first with the amount of grease used present on the bare lever. The uncertainty concerning the spring constant $k$ will be a limitation at these micrometer scales. For accurate and absolute measurements of submicrogramm samples a calibration with a known reference mass is recommended, especially to determine $k$ precisely. The mass of this test sample is rather large if one observes the frequency shift. In principle, shifts smaller than $50 \mathrm{~Hz}$ can easily be detected. They correspond to a very low limit for the mass detection of about $0.3 \mathrm{ng}$.

\section{Example of measurement}

For demonstration purposes, an angular-dependent measurement was performed on the same Hg-1201 single crystal as described above. As for most high- $T_{c}$ cuprates, this sample shows a large anisotropy due to its layered structure. Usually, the $a b$-plane of the sample is defined by the $\mathrm{CuO}_{2}$ layers and the $c$-axis is perpendicular to this plane. The magnetic field $\vec{B}$ is rotated about the $b$-axis in order to measure the $a c$-anisotropy. A typical torque curve is presented in Fig. 9. At $\theta=90^{\circ}$, the magnetic field crosses the $a b$-plane of the sample and the torque curves show irreversibility owing to vortex pinning around this angle. By increasing the magnetic field and/or temperature, the torque signal becomes fully reversible. In this case, it is possible to analyze such a curve using a model for three-dimensional anisotropic superconductors and a fitting equation derived by Kogan. ${ }^{21}$ Such a procedure allows the three superconducting parameters of interest, $\gamma, \lambda_{a b}$, and $H_{c 2}^{c}$, to be determined, where $\gamma=\left(m_{c}^{*} / m_{a b}^{*}\right)^{1 / 2}$ is the effective mass anisotropy, $\lambda_{a b}$ the 


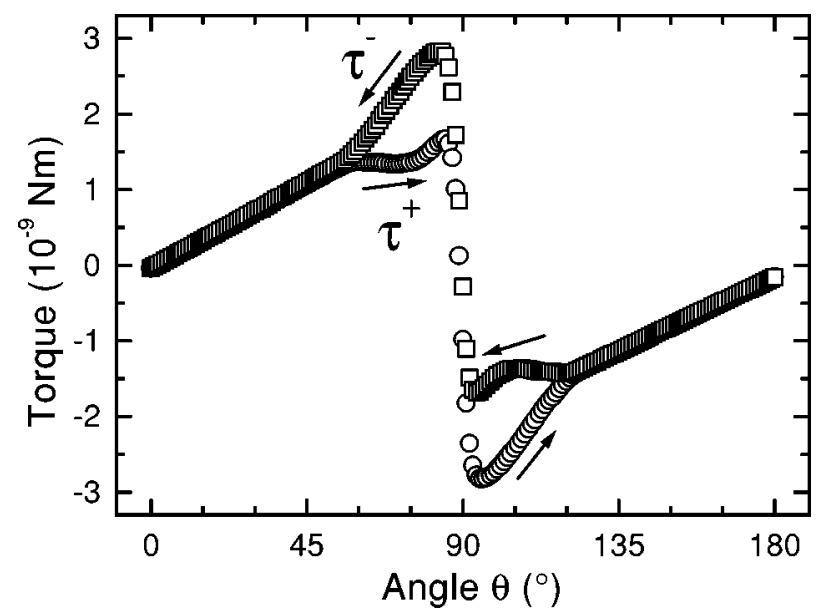

FIG. 9. Example of angular-dependent torque measured on a high- $T_{c} \mathrm{Hg}-$ 1201 single crystal. $\theta=90^{\circ}$ for $\vec{B}$ parallel to the $a b$-plane of the sample.

in-plane penetration depth, and $H_{c 2}^{c}$ the upper critical field in $c$-direction. In Fig. 9 the output signal of the Wheatstone bridge is directly calibrated in torque units. We see that below and above the $a b$-plane crossing, the torque signal can become rather significant. In large signal limits the deformation of the lever itself is no longer negligible but yields a small sample orientation change. In our example the reference direction is the $c$-axis $\left(\theta=0^{\circ}\right)$ of the crystal, which follows the cantilever deflection. Thus the orientation of the magnetic field $\vec{B}$ must be measured relative to this moving reference and not to a fixed reference in the laboratory, as is usually done. This requires a small angular correction factor, which can be estimated to be $5.3 \times 10^{-4} \% / \mathrm{pNm}$ and $3.5 \times 10^{-4} \% / \mathrm{pNm}$ for the two-leg and the three-leg cantilever, respectively. The sign of the correction depends on whether the sample is diamagnetic or paramagnetic. In the case of our Hg-1201 crystal, the two peaks of the typical Kogan-like signal are shifted closer together after angle correction. Consequently the three superconducting parameters deduced from a fit will be modified. In particular the correction would lead to significantly higher $\gamma$ values. To avoid obtaining misleading results it is thus important to take this angular correction into account when dealing with samples with large torque signals.

\section{E. Further applications}

We have shown that, for superconducting samples, a torquemeter can be calibrated by using the Meissner slope. ${ }^{9}$ The magnetization $M$ in the Meissner state is linearly related to the applied magnetic field $H$ by $M=-H(1-N)^{-1}$, where $N$ is the demagnetization factor. ${ }^{22}$ The calibration is not always straightforward because, for a sample with a complex shape, the demagnetization factor can be difficult to determine. This method is not optimal for the calibration of very flat samples (films) with a demagnetization factor close to 1 . In fact a useful application of the integrated calibration loop is the precise determination of $N$ for a complicated sample shape, if one can assume a Meissner fraction of $100 \%$. Regarding this problem from another angle, if the demagneti- zation factor is well known, the use of both Meissner slope and calibrating loop allows one to extract the sample's Meissner fraction.

The main advantage of using a torquemeter is the ability to obtain angular-dependent measurements of anisotropic samples in a very simple way. For specific samples, it may be interesting to measure the torque anisotropy with respect to different axes. With standard systems, it has been necessary to change the sample orientation relative to the torquemeter itself. This means warming up and opening the system to reposition the sample. By mounting our new lever on a small rotator or goniometer, in situ angular-dependent measurements of an anisotropic sample with respect to different axes become possible. This procedure does not reorient the sample alone, which is rather difficult for microscopic samples, but the entire cantilever with the sample on it.

\section{CONCLUSIONS}

The cantilevers presented here are powerful tools for torque magnetometry. They can work in the flexion and torsion modes and consequently are suitable for measuring a torque with respect to two axes. Without taking special precautions, one can use the torquemeter to reach for both axes a typical resolution of $10^{-12} \mathrm{Nm}$ in the static regime and $10^{-14} \mathrm{Nm}$ in the dynamic regime. In one version of the levers, a small current loop has been integrated. It allows an absolute, quick, and precise calibration of the torquemeter in flexion as well as in torsion mode. This calibration is essential for a direct comparison between data obtained in flexion and torsion mode, because the torque sensitivity for each mode is usually not the same. We also fabricated a second version without a calibration loop but with a slightly higher torque sensitivity. The calculations of the levers' properties are in excellent agreement with their experimental behavior.

The range of applications of such sensors is appreciable in the domain of magnetometry. Their very high sensitivity and stability, particularly in the dynamic regime, allow investigations of very small samples. In the future, it will be possible to implant submicrostructures in ordered or disordered arrays directly on the lever platform itself. Another use of the calibration loop is to drive the lever with a feedback circuit in order to make it artificially more rigid. Such a device without a sample can also be applied to measure directly an external field (gaussmeter). The resonance frequencies of these devices are rather large $(>50 \mathrm{kHz})$. Consequently, their fast response allows the detection of short relaxation effects or magnetization measurements in pulsed magnetic fields.

Although we demonstrated the potential of these cantilevers for torque magnetometry, the design does not exclude general uses such as for force or displacement detection.

\section{ACKNOWLEDGMENTS}

The authors are pleased to acknowledge the contributions of and discussions with our colleagues C. Andreoli, U. Drechsler, A. Gasser, and M. Tschudy of the IBM Microand Nanomechanics group. They thank M. Delobelle of the 
University of Franche-Comté in Besançon for information on single crystal silicon. The Hg-1201 single crystal was provided by J. Karpinski. They are grateful to the Ecole d'Ingénieurs du Canton de Neuchâtel (EICN) in Le Locle for technical support in the domain of micromanipulation. They also acknowledge valuable discussions with members of the Institut de Microtechnique (IMT) and of the Center Suisse d'Electronique et de Microtechnique (CSEM) in Neuchâtel. This work was partially supported by the Swiss National Foundation.

\section{APPENDIX}

The calculations for a two-leg and a three-leg lever are very similar. Equations (2) and (3) can be recalculated for the three-leg lever. The averaged stress over the piezoresistive path for the flexion mode becomes

$$
\overline{\sigma_{p}^{f}}=\frac{2 \tau_{y}}{(w-p) t^{2}}
$$

and the corresponding stress for the torsion mode

$$
\overline{\sigma_{p}^{t}}=\frac{3}{2} \tau_{x}\left(\frac{b(w-p) t^{2}}{l}+\frac{G l}{E b}\left[\frac{\left(w^{3}-p^{3}\right)+(w-p) t^{2}}{2}\right]\right)^{-1} .
$$

All quantities appearing in these equations are the same as described above.

${ }^{1}$ J. G. E. Harris, D. D. Awschalom, K. D. Maronowski, and A. C. Gossard, Rev. Sci. Instrum. 67, 3591 (1996).

${ }^{2}$ G. Meyer and N. M. Amer, Appl. Phys. Lett. 53, 1045 (1988).

${ }^{3}$ S. Alexander, L. Hellemaus, O. Marti, J. Scheir, V. Ealings, P. K.
Hausma, M. Longmire, and J. Gurley, J. Appl. Phys. 65, 164 (1989).

${ }^{4}$ M. Tortonese, H. Yamada, R. C. Barrett, and C. F. Quate, in Proceedings of Transducers 1991 (IEEE, New York, 1991), 91CH2817-5, p. 448; M. Tortonese, R. C. Barrett, and C. F. Quate, Appl. Phys. Lett. 62, 834 (1993).

${ }^{5}$ F. J. Giessibl and B. M. Trafas, Rev. Sci. Instrum. 65, 1923 (1994).

${ }^{6}$ T. Itoh and T. Suga, in Proceedings of Transducers 1993, 7th International Conference on Solid-State Sensors and Actuators (IEEJ, Tokyo, 1993), p. 610.

${ }^{7}$ N. Blanc, J. Brugger, N. F. de Rooij, and U. Dürig, J. Vac. Sci. Technol. B 14, 901 (1996).

${ }^{8}$ R. Berger, Ch. Gerber, and J. K. Gimzewski, Microelectron. Eng. 35, 373 (1997).

${ }^{9}$ C. Rossel, P. Bauer, D. Zech, J. Hofer, M. Willemin, and H. Keller, J. Appl. Phys. 79, 8166 (1996).

${ }^{10}$ D. Zech, J. Hofer, C. Rossel, P. Bauer, H. Keller, and J. Karpinski, Phys. Rev. B 53, R6026 (1996).

${ }^{11}$ D. E. Farrell, Phys. Rev. B 51, 9148 (1995).

${ }^{12}$ R. Griessen, M. J. G. Lee, and D. J. Stanley, Phys. Rev. B 16, 4385 (1977).

${ }^{13}$ M. Qvarford, K. Heeck, J. G. Lensink, R. J. Wijngaarden, and R. Griessen, Rev. Sci. Instrum. 63, 5726 (1992).

${ }^{14}$ H. G. Schnack, R. Griessen, P. Noordeloos, and K. Heeck, Physica C 266, 285 (1996).

${ }^{15} \mathrm{M}$. Willemin and C. Rossel (unpublished).

${ }^{16}$ Y. Kanda, Sens. Actuators A 28, 83 (1991).

${ }^{17}$ S. M. Sze, Physics of Semiconductor Devices (Wiley, New York, 1981).

${ }^{18}$ C. Bourgeois, J. Hermann, N. Blanc, N. F. de Rooij, and F. Rudolf, in Transducers 1995, Proceedings of 8th International Conference on SolidState Sensors and Actuators, and Eurosensors IX (Elsevier, Amsterdam, 1995), pp. 92-95.

${ }^{19}$ R. A. Buser, Ph.D. dissertation, Institut de Microtechnique de l'Université de Neuchâtel, Switzerland, June 1989.

${ }^{20}$ J. Brugger, M. Willemin, C. Rossel, G. Beljakovic, and P. Vettiger, in Proceedings of Transducers 97, Chicago, IL, June 16-19, 1997 (IEEE, Piscataway, 1997), p. 419.

${ }^{21}$ V. G. Kogan, Phys. Rev. B 38, 7049 (1988).

${ }^{22}$ J. A. Osborn, Phys. Rev. 67, 351 (1945). 